\section{Sindrome Metabólica: Ainda Indefinida, Mas Útil na Identificação do Alto Risco Cardiovascular}

$\mathbf{N}$ OS Últimos ANOS, NO BRAsil e em países do mundo desenvolvido, a obesidade se tornou um problema de saúde pública mais importante que a desnutrição. Com o aumento de prevalência de obesidade, identificou-se a relação entre gordura visceral abdominal e resistência à insulina, e uma nova entidade clínica heterogênea associada com obesidade abdominal e resistência à insulina, foi identificada como um fator de risco maior para doença cardiovascular aterosclerótica.

Esta entidade, inicialmente denominada "síndrome X" por Gerald Reaven ou "síndrome de resistência à insulina" por outros, é agora conhecida como síndrome metabólica. Embora as tentativas iniciais de definir a síndrome tenham levado a ampla discrepância com respeito aos critérios diagnósticos, as definições atuais proporcionam uma maneira útil e prática de se identificar indivíduos com risco aumentado de desenvolver diabetes mellitus do tipo 2 (DM2), doença cardiovascular aterosclerótica e morte cardiovascular.

Nos últimos anos, dois sistemas de classificação ou critérios diagnósticos para a síndrome metabólica vêm sendo amplamente utilizados. Eles apresentam similaridade quanto aos fatores de risco cardiovasculares, incluindo obesidade abdominal, intolerância à glicose / resistência à insulina, dislipidemia e hipertensão arterial. Em abril de 2005 a International Diabetes Federation (IDF) reformulou o sistema de Classificação da NCEP-ATP III, apresentando critérios mais estritos para o diagnóstico de síndrome metabólica. Todos estes critérios deverão ser validados nos próximos anos, mas o da ATP III e o da IDF, são os de mais fácil aplicação clínica.

Os componentes individuais da síndrome metabólica são fatores de risco independentes para o desenvolvimento de doença cardiovascular aterosclerótica. As tentativas de se estabelecer critérios diagnósticos para esta síndrome são baseadas no princípio de que estes componentes podem agir de maneira sinérgica ou aditiva amplificando o risco, o que ainda não foi demonstrado. Entretanto, deve ser mencionado que os estudos sobre mecanismos fisiopatológicos e riscos cardiovasculares, bem como as tentativas de definição da síndrome metabólica, são recentes e ainda restam muitas dúvidas e indefinições sobre o assunto.

Embora haja uma clara associação com a resistência à insulina, este parece não ser único mecanismo envolvido na fisiopatologia da síndrome metabólica. Não é raro estabelecermos o diagnóstico de resistência à insulina sem que estejam presentes os componentes que caracterizam a síndrome. Mecanismos fisiopatológicos que determinam o acúmulo de gordura visceral e maior grau de lipólise estão também provavelmente e intimamente envolvidos no desenvolvimento da síndrome metabólica. As questões ainda são tantas que alguém, brincando e lembrando Reaven, já mencionou que o nome mais apropriado seria Xíndrome Metabólica.

Uma revisão recente do tópico aprovada pelo Comitê de Prática Profissional da Associação Americana de Diabetes e também por um

\section{editorial}

\author{
Mario J.A. Saad \\ Maria Teresa Zanella \\ Sandra Roberta G. Ferreira
}

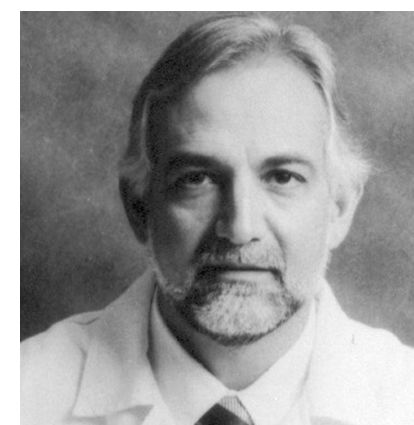

Professor Titular de Clínica Médica da Faculdade de Ciências Médicas da Universidade Estadual de Campinas, SP (UNICAMP)

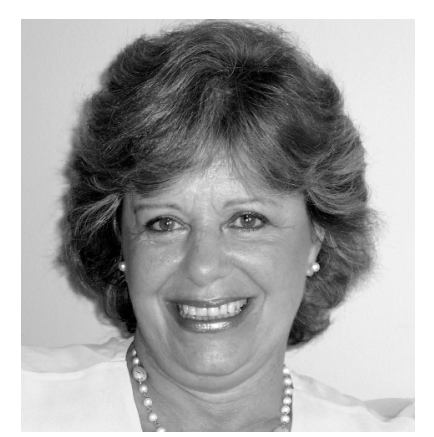

Professora Titular da Disciplina de Endocrinologia da Universidade Federal de São Paulo (UNIFESP) e Presidente do Departamento de Aterosclerose e Dislipidemia da Sociedade Brasileira de Endocrinologia e Metabologia (SBEM)

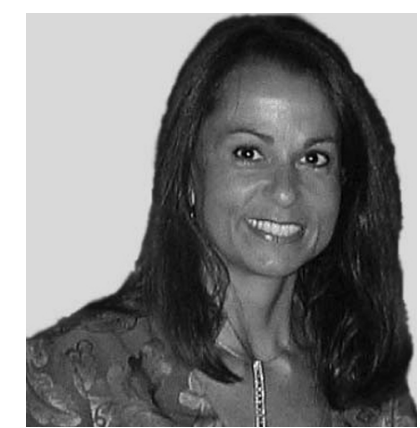

Professora Titular do Departamento de Nutrição da Faculdade de Saúde Pública da Universidade de São Paulo (FSP-USP) 
Comitê da Associação Européia para o Estudo do Diabetes apontou questões pertinentes em relação à síndrome metabólica, descritas a seguir.

1) Os critérios diagnósticos são ambíguos ou incompletos, e a base racional para a escolha de limiares é mal definida;

2) O valor de se incluir diabetes na definição é questionável;

3) A resistência à insulina como hipótese etiológica unificadora (que caracterizaria o aglomerado de fatores de risco como síndrome) é incerta;

4) Não há uma razão clara para se incluir ou excluir outros fatores de risco cardiovasculares;

5 ) O risco cardiovascular associado à síndrome parece não ser maior que a soma das partes (cada fator de risco);

6) O tratamento da síndrome não é diferente do tratamento de cada um de seus componentes; 7) $\mathrm{O}$ valor médico do diagnóstico da síndrome não é claro.

Essas dúvidas são relevantes, e ajudam a levantar questões que precisam ser respondidas em novos estudos, ou através da re-análise de dados já existentes. Entretanto, os questionamentos sobre critérios diagnósticos, a busca de novos mecanismos fisiopatológi$\cos$ e a ausência de um tratamento específico são motivos para se aprofundar as investigações, e não para negar a existência da síndrome. A possível identificação de novos componentes etiopatogênicos, deverá se somar ou complementar a resistência à insulina, e não reduzir sua importância na síndrome metabólica. É importante reconhecer que a síndrome metabólica representa um avanço clínico na prevenção de doenças cardiovasculares, independente da ausência de sinergismo no risco individual de cada componente, porque o agrupamento desses fatores e o estabelecimento de critérios sistematizam a prática clínica, e induzem o médico a pensar coletivamente nestes fatores de risco.

Neste número especial dos ABE\&M sobre a Síndrome Metabólica que tivemos a grata oportunidade de editar, tivemos excelentes contribuições de cientistas da área básica e de clínicos experientes que, temos certeza, serão de grande valia para podermos continuar acompanhando todos os avanços do conhecimento nesta área. Assim, gostaríamos de agradecer a todos os colaboradores que aceitaram prontamente o nosso convite e também ao nosso amigo e grande Editor-Chefe, Prof. Dr. Claudio Elias Kater que confiou a nós esta importante tarefa.

\section{Endereço para correspondência:}

Maria Teresa Zanella

Centro Integrado de Metabologia Cardiovascular

Universidade Federal de São Paulo

Rua Leandro Dupret, 365 - Vila Clementino

04025-011 São Paulo, SP 